\title{
Dosimétrie neutronique par réaction $(n, p)$ et utilisation de détecteurs polymériques
}

\author{
A. CHAMBAUDET*, D. FELLMANN**, P. LE THANH ${ }^{\star \star \star}$ et \\ C. VUILLEUMIER*
}

(Manuscrit reçu le 21 janvier 1987)

\begin{abstract}
RÉSUMÉ
Après une réaction de type $(n, p)$, la trajectoire des protons est visualisée dans les détecteurs solides de traces permettant ainsi la dosimétrie des neutrons incidents. Les détecteurs utilisés (LR115 et CR39) sont de type polymérique et possèdent une grande sensibilité aux protons. Les faibles épaisseurs du LR115 $(7,2$ et $8,2 \mu \mathrm{m})$ permettent après développement électro-chimique des traces nucléaires un comptage rapide par la méthode des décharges électriques. Le comptage sur CR39, d'épaisseur plus importante, est réalisé par analyse automatique d'image (Quantimet $\mathbf{7 2 0}$ ). La réponse par unité d'équivalent de dose décroít lentement en fonction de l'énergie croissante des neutrons. La mise en œuvre des systèmes rapides de comptage permet d'envisager l'utilisation de ces détecteurs en routine.
\end{abstract}

\section{ABSTRACT}

After an $(n, p)$ type of reaction, the trajectory of protons is visualized in solid track detectors, thus permitting the dosimetry of incident neutrons. The detectors used (LR115 and CR39) are of a polymetric type having great sensitivity to protons. The relative thinness of the LR115 samples $(7.2$ and $8.2 \mu \mathrm{m})$ allows rapid counting using the electric discharge method after electrochemical development of nuclear tracks. Counting on the thicker CR39 is carried out by automatic image analysis (Quantimet 720 ). The response per equivalent dose unit slowly decreases as a function of the increasing energy of neutrons. The rapid counting systems permit the use of these detectors for routine measurements.

- Laboratoire de microanalyses nucléaires, UER Sciences, La Bouloie, 16, route de Gray. 25030 Besançon Cedex (France).

** Laboratoire de cytologie et de cytogénétique, CHU, place Saint-Jacques, 25030 Besançon Cedex (France).

*** CEN Saclay, SPR, 91191 Gif-sur-Yvette (France). 


\section{INTRODUCTION}

A l'heure actuelle, il n'existe pas de dosimètre individuel neutronique dont la réponse soit constante quelle que soit l'énergie des neutrons incidents. L'un des problèmes de la dosimétrie personnelle étant la mesure des neutrons d'énergie inférieure à $1 \mathrm{MeV}$, des tentatives ont été réalisées avec des dosimètres plastiques à convertisseurs au bore et au lithium donnant des réactions $(n, \alpha)$ ou des dosimètres à convertisseurs fissiles.

Dans cette optique, il paraît intéressant d'exploiter les réactions (n,p) obtenues avec des convertisseurs de polyéthylène et de détecter les protons émis dans un matériau polymérique.

Le détecteur le plus utilisé est le CR39. C'est un détecteur solide de traces nucléaires de type polymérique, qui présente une grande sensibilité aux protons de faibles énergies. Du fait de son épaisseur importante $(>400$ $\mu \mathrm{m}$ ), le développement chimique ou électrochimique de ce détecteur sera suivi d'un comptage optique ou d'un comptage automatique par analyse d'image. En effet, les traces développées ne peuvent pas traverser entièrement le détecteur pour permettre le dépouillement rapide par la méthode des décharges électriques.

Nous proposons donc d'utiliser un autre détecteur solide de traces nucléaires, le LR115, comme détecteur de protons. Egalement de nature polymérique, ce film présente l'avantage d'être commercialisé en faible épaisseur, ce qui permet, après le développement chimique ou électrochimique, d'utiliser le comptage par décharges disruptives.

\section{Réactions (n,p) avec les constituants du tissu mou}

La figure 1 montre la courbe de dose de première collision pour différents constituants du tissu mou en fonction de l'énergie des neutrons. Pour les neutrons thermiques, la réaction ${ }^{14} \mathrm{~N}(n, p){ }^{14} \mathrm{C}$ est d'une importance particulière dans le tissu humain. Elle produit un proton de $0,58 \mathrm{MeV}$, quantité d'énergie qui sera absorbée localement. Au-dessus de $1 \mathrm{keV}$, la majeure partie de la dose absorbée est due à la diffusion élastique avec l'hydrogène engendrant des protons de recul.

Dans les réactions de diffusion $(n, p)$ dues aux neutrons d'énergies élevées, les produits de réaction peuvent avoir différentes énergies dépendant de l'énergie engagée et de l'angle d'émission des particules.

L'enregistrement des protons de recul dans les détecteurs solides de traces apparaît comme étant l'une des méthodes intéressantes dans la conception d'un dosimètre efficace de neutrons rapides. II est alors nécessaire d'utiliser un convertisseur riche en hydrogène ou équivalent du tissu humain. Le polyéthylène $\left(\mathrm{CH}_{2}\right)_{n}$, très hydrogéné, est choisi comme convertisseur dans nos ensembles convertisseur-détecteur. 


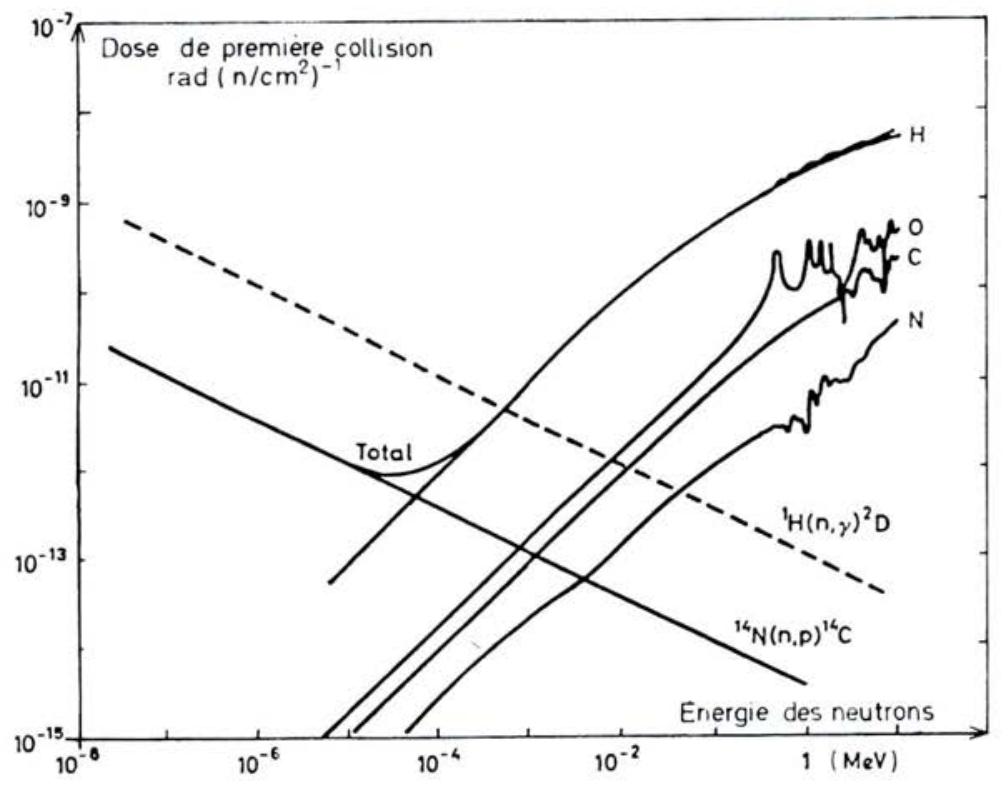

Fig. 1. - Courbe de dose de première collision calculée pour le tissu mou en fonction de l'énergie des neutrons, d'après [5].

\section{Les détecteurs solides de traces LR115 et CR39}

De formule brute $\left(\mathrm{C}_{6} \mathrm{H}_{2} \mathrm{O}_{9} \mathrm{~N}_{2}\right)$, le nitrate de cellulose apparaît comme l'un des matériaux polymériques les plus sensibles du point de vue de la détection des particules chargées. Parmi ses multiples avantages, il est insensible aux rayonnements $\beta, \gamma$ et à la lumière du jour. Commercialisé sous le nom de LR115 (par la société Kodak), il est constitué d'une pellicule de nitrate de cellulose teintée rouge (6 à $13 \mu \mathrm{m}$ d'épaisseur) déposée sur un support de polyester de $100 \mu \mathrm{m}$ d'épaisseur.

Après développement chimique (DC), les traces qui traversent le détecteur apparaissent en microscopie optique sous forme de taches lumineuses blanches sur fond rouge.

Un autre détecteur de traces nucléaires, le carbonate allyldiglycol connu sous le nom commercial de columbia resin (CR39), a pour formule brute $\left(\mathrm{C}_{12} \mathrm{H}_{18} \mathrm{O}_{7}\right)$. II est transparent, amorphe et hautement sensible aux particules lourdes chargées. Son épaisseur varie, suivant les fabrications, d'une centaine de micromètres à un millimètre. 


\section{Le développement électrochimique (DEC)}

Les traces latentes développables de protons produits par les neutrons ètant très courtes (quelques micromètres), le développement électrochimique permet de les conserver. Le DEC exploite l'action combinée d'un réactif chimique et d'un champ électrique alternatif élevé permettant d'engendrer des phénomènes d'avalanches le long de la zone de dommages latents. Pour ce type de développement, le détecteur est placé entre deux cellules de polymétacrylate de méthyle contenant l'électrolyte (fig. 2). La tension alternative est appliquée à travers le détecteur à l'aide de deux électrodes immergées dans l'électrolyte. Au cours du développement, on observe deux étapes:

- Agrandissement de la trace: la condition pour qu'une trace soit agrandie par le réactif chimique est que la composante normale à la surface du détecteur de la vitesse d'attaque chimique le long de la trace soit supérieure à la vitesse d'attaque chimique dans les parties non endommagées;

- Formation de l'arborescence: la zone endommagée caractérisant la trace latente se remplit d'électrolyte; à l'extrémité de la trace, le champ électrique devient très élevé et peut alors initier des fissurations dans le matériau détecteur non endommagé et conduire ainsi à la formation de l'arborescence.

Ces phénomènes électriques combinés à l'attaque chimique produisent donc des dommages qui se propagent au travers de la pellicule de nitrate de cellulose en générant alors une perforation ("trou") (fig. 3), permettant un comptage ultérieur par décharges disruptives.

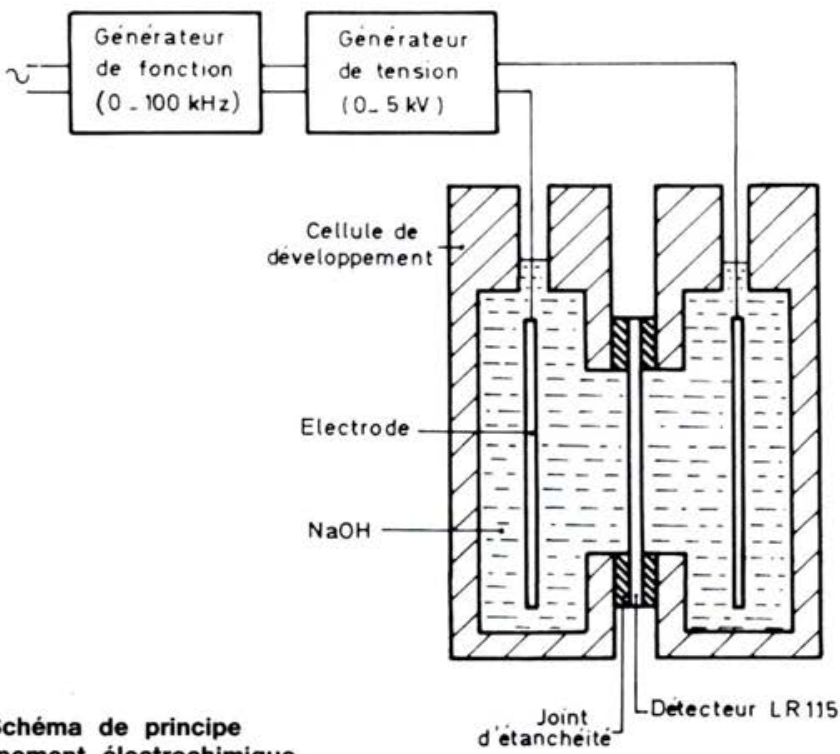

Fig. 2. - Schéma de principe du développement électrochimique. 
DEVELOPPEMENT CHIMIQUE

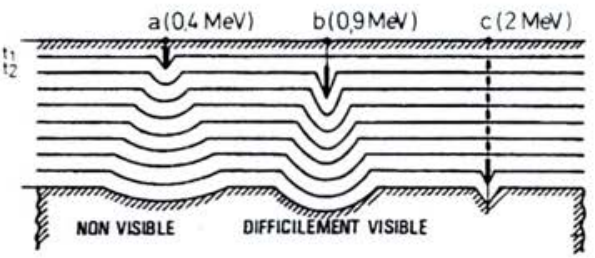

DEVEL OPPEMENT ELECTRO-CHIMIQUE

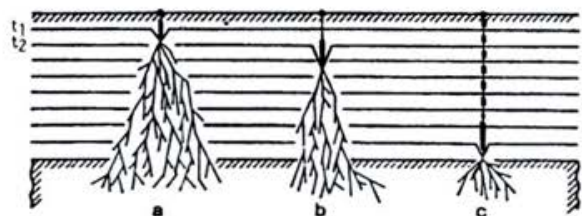

SURFACE INITIALE

SURFACE DEVELOPPEE

SURFACE INTIALE

SURFACE DEVELOPPEE

Fig. 3. - Formation des traces à différents temps de développement respectivement par développement chimique (partie supérieure)

et développement électrochimique (partie inférieure).

\section{Comptage des traces par la méthode à décharges disruptives}

Cette méthode est rapide et précise pour les faibles densités de trous $\left(<10^{3}\right.$ trous $\left./ \mathrm{cm}^{2}\right)$ contrairement au comptage optique qui est très long et fastidieux mais qui est cependant indispensable en tant que référence.

Lors de ce comptage, le détecteur solide de traces comportant des trous est utilisé comme le diélectrique d'un condensateur plan dont les armatures sont constituées par deux feuilles de mylar aluminisé. Une haute tension appliquée aux bornes provoque une décharge à travers le trou en vaporisant l'aluminium au regard de celui-ci. Cette décharge crée une variation de la tension aux bornes du circuit électrique qui est amplifiée, discriminée et comptée (fig. 4). La reproductibilité de ce comptage est très satisfaisante pour de faibles densités mais, par contre, une perte de comptage est inévitable pour les densités de trous plus élevées.
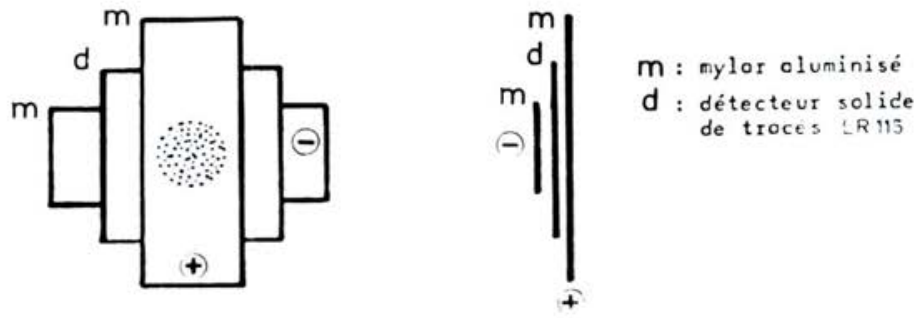

Fig. 4. - Dispositions du film lors du comptage par décharge électrique. Les feuilles de mylar aluminisées sont disposées de telle sorte que les faces aluminisées soient en regard.

VOL. $22-\mathrm{N}^{\circ}{ }_{4}$ 
Cette méthode de comptage s'applique au détecteur LR $\uparrow 15$ dont la couche polymérique sensible de quelques $\mu \mathrm{m}$ d'épaisseur peut être prélevée de son support après développement. II n'en est pas de même pour le CR39 dont l'épaisseur trop importante ne permet pas la mise en œuvre de cette technique. II faut alors recourir pour le dénombrement des traces à la microscopie optique classique, encore une fois longue et fastidieuse, ou faire appel à des techniques très performantes d'analyse d'image.

\section{Comptage des traces par analyse d'image}

L'analyseur d'image utilisé ici est le Quantimet 720 de chez Cambridge dont le fonctionnement est programmé par le biais d'un ordinateur (language Fortran 4). L'analyse est basée sur une analyse optique, réalisée à partir d'un microscope relié à une caméra vidéo qui transmet au module d'entrée un signal permettant aux différents modules de l'analyseur de traiter une image digitalisée. Cette dernière est composée de 630000 points images (picture elements) que l'on peut affiner tout en observant les modifications sur écran vidéo. On dispose de 64 niveaux de gris. On peut ainsi déterminer sur un échantillon donné le niveau de gris correspondant aux éléments à comptabiliser qui deviendra la valeur seuil. L'analyseur prendra en compte tous les éléments "plus blancs que" ou "plus noirs que". Dans le cas de l'analyse de traces nucléaires dans nos films détecteurs polymériques, la qualité exceptionnelle des contrastes permet une mise au point efficace (contraste blanc sur fond rouge pour le LR115, contraste noir sur fond incolore pour le CR39). Le dénombrement des événements enregistrés est déduit d'une trace révélée en tenant compte des recouvrements éventuels.

\section{Détection des alpha de faible énergie au moyen du nitrate de cellulose}

D'après Decossas [1, 2] et Lück [7], le nitrate de cellulose n'enregistre les traces de protons que si ces derniers ont une énergie inférieure à 0,7 $\mathrm{MeV}$. Le détecteur LR115 permet donc cet enregistrement et les traces révélées obtenues peuvent être comptées, en plus de la technique citée précédemment, par la méthode des décharges disruptives et permettre ainsi de comparer les efficacités de comptage obtenues par analyse d'image et par décharges disruptives.

Le parcours développable des protons dans le LR115 n'est que de quelques $\mu \mathrm{m}$; c'est pourquoi, afin de pouvoir déterminer les différentes caractéristiques du détecteur à utiliser (épaisseur et durée de développement optimales), il est plus aisé, dans un premier temps, de substituer les protons par des particules alpha de faible énergie ayant à peu près le même parcours développable dans le nitrate de cellulose. Les particules alpha incidentes de $0,4 \mathrm{MeV}$ sont obtenues à l'aide d'une source alpha d'américium 241 en interposant un ralentisseur de makrofol de $34 \mu \mathrm{m}$. Les détecteurs sont alors irradiés sous vide (à $10 \mathrm{~cm}$ de la source).

Les différents paramètres du développement électrochimique sont la concentration du réactif chimique, le voltage appliqué, la fréquence, la température. Une solution de soude, un champ alternatif de $4 \mathrm{~V} / \mu \mathrm{m}$ et une fréquence de $500 \mathrm{~Hz}$ permettent d'obtenir les meilleurs résultats. II faut, en fait, 
déterminer une température de développement qui tienne compte du temps de développement et de la diminution de l'épaisseur du détecteur par l'attaque chimique.

La figure 5 montre pour le LR115 de $8,2 \mu \mathrm{m}$ d'épaisseur initiale, l'efficacité de comptage en fonction du temps de développement à différentes températures $\left(45^{\circ} \mathrm{C} ; 50^{\circ} \mathrm{C} ; 55^{\circ} \mathrm{C}\right)$ avec une solution de soude à $10 \%$. L'efficacité de comptage par décharges électriques est définie comme étant le rapport du nombre de trous comptés au nombre total de traces et trous évalué par le comptage optique, c'est-à-dire le nombre de particules alpha incidentes. Dans la perspective d'un comptage par décharges, les conditions optimales sont l'utilisation d'une solution de soude à $10 \%$ en poids, une température de $45^{\circ} \mathrm{C}$ pendant $7 \mathrm{~h} 30 \mathrm{~min}$ en appliquant un champ de $4 \mathrm{~V} / \mu \mathrm{m}$ et une fréquence de $500 \mathrm{~Hz}$ (photo 1).

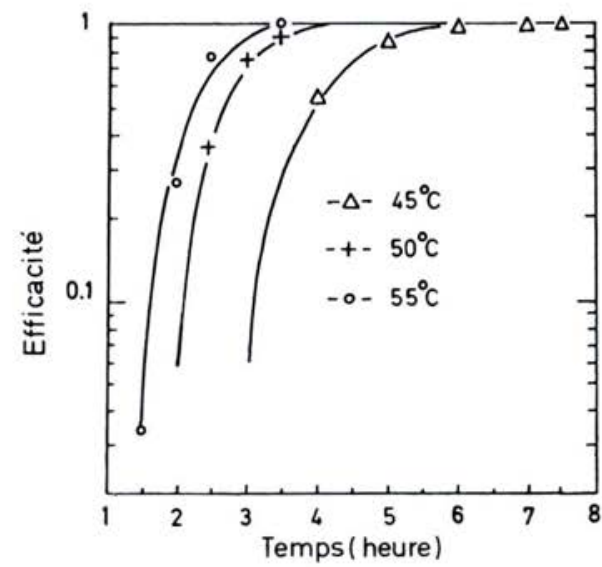

Fig. 5. - Efficacité de détection en fonction du temps de développement à différentes températures $\left(45^{\circ} \mathrm{C}, 50^{\circ} \mathrm{C}, 55^{\circ} \mathrm{C}\right)$ pour le LR115 d'épaisseur initiale $8,2 \mu \mathrm{m}$.

La photo 2 montre ces mêmes traces développées dans les mêmes conditions en absence de champ électrique: il apparaît nettement que les traces ne traversent pas le détecteur. En conséquence, le comptage par décharges disruptives ne peut pas être utilisé.

Les résultats de développement ainsi obtenus sont alors vérifiés avec des protons de $0,58 \mathrm{MeV}$ issus de la réaction ${ }^{14} \mathrm{~N}(n, p){ }^{14} \mathrm{C}$.

VOL. $22-\mathrm{N}^{\circ} 4$ 


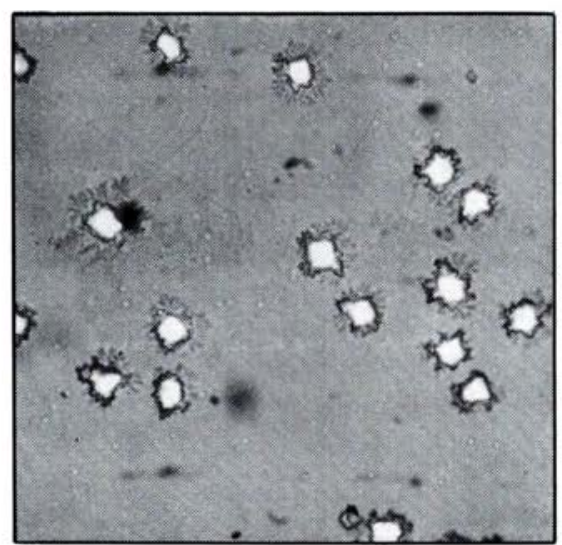

Photo 1. - Particules alpha de 0,4 MeV révélées dans le LR115 $(8,2 \mu \mathrm{m})$ par développement électrochimique (10\% $\mathrm{NaOH}, 45^{\circ} \mathrm{C}$. $400 \mathrm{~V}, 500 \mathrm{~Hz}$ ).

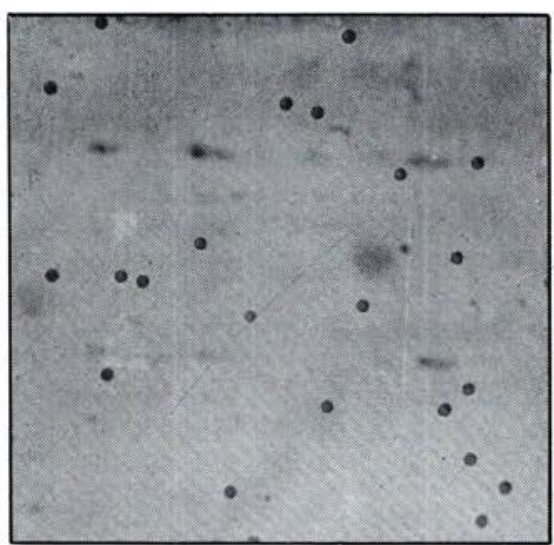

Photo 2. - Mêmes traces après développement chimique (10\% $\mathrm{NaOH}, 45^{\circ} \mathrm{C}$ ).

\section{Détection de protons avec le LR115}

La réaction ${ }^{14} \mathrm{~N}(n, p){ }^{14} \mathrm{C}$ mise en jeu est similaire à celle qui se produit dans les tissus humains. L'azote de l'air et l'azote contenu dans le nitrate de cellulose jouent le rôle de convertisseurs. Les neutrons utilisés sont des neutrons froids fournis par le réacteur "Orphée" (CEN Saclay). Un écran de cadmium $(0,5 \mathrm{~mm})$ avec un trou de $1,13 \mathrm{~cm}^{2}$ est placé devant et contre le détecteur et permet de délimiter le faisceau de neutrons et par conséquent

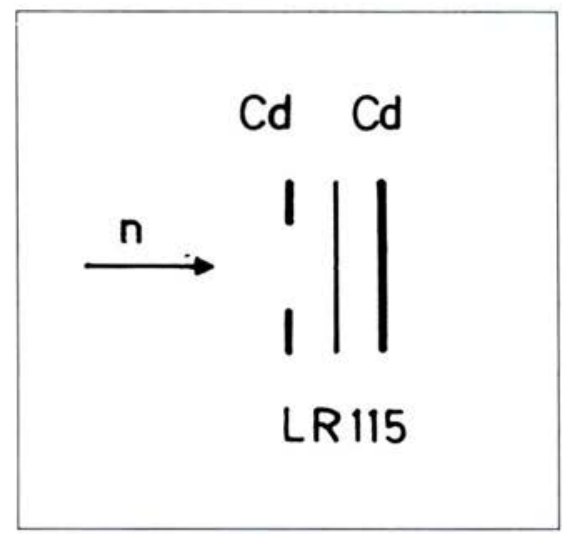

Fig. 6. - Représentation schématique du dispositif expérimental d'irradiation aux neutrons thermiques.

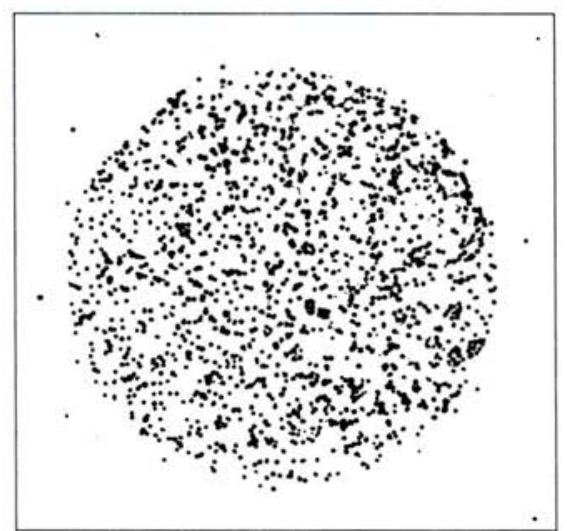

Fig. 7. - Réplique en aluminium après comptage par décharges électriques. 


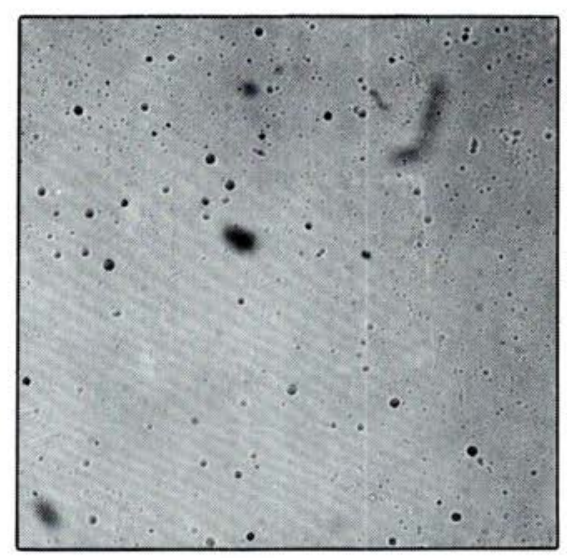

Photo 3. - Traces de protons issus de

la réaction ${ }^{14} \mathrm{~N}(n, p){ }^{14} \mathrm{C}$ dans le détecteur LR115 développé chimiquement $\left(10 \% \mathrm{NaOH}, 45^{\circ} \mathrm{C}\right)$.

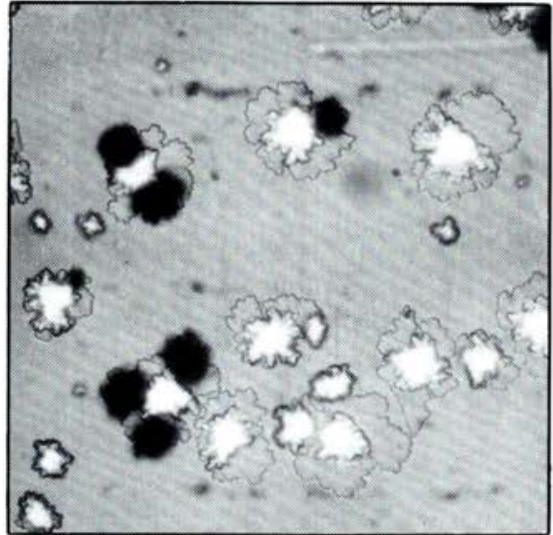

Photo 4. - Traces de protons dans le détecteur LR115 développé électrochimiquement $\left(10 \% \mathrm{NaOH}, 45^{\circ} \mathrm{C}\right.$, $400 \mathrm{~V}, 500 \mathrm{~Hz}$ ).

de protons (fig. 6 et 7 et photos 3 et 4) [6]. Le cercle obtenu prouve sans ambiguilté que les traces sont bien dues aux protons.

Les conditions optimales sont vérifiées avec les détecteurs LR115 d'une épaisseur initiale de 8,2 et $7,2 \mu \mathrm{m}$ en faisant varier le temps de développement (tableau I).

\section{TABLEAU ।}

Efficacité de détection des protons issus de la réaction ${ }^{14} \mathrm{~N}(\mathrm{n}, \mathrm{p}){ }^{14} \mathrm{C}$ au moyen de détecteurs LR115 de 7,2 et $8,2 \mu \mathrm{m}$ d'épaisseur.

(conditions du développement électrochimique: solution de soude à $10 \%$, $45^{\circ} \mathrm{C}, 400$ V, $500 \mathrm{~Hz}$ )

\begin{tabular}{|c|c|c|c|c|}
\hline $\begin{array}{c}\text { Epaisseur } \\
\text { initiale } \\
(\mu \mathrm{m})\end{array}$ & $\begin{array}{c}\text { Temps de } \\
\text { développement }\end{array}$ & $\begin{array}{c}\text { Epaisseur } \\
\text { finale } \\
(\mu \mathrm{m})\end{array}$ & $\begin{array}{c}\text { Densité de } \\
\text { trous } \\
\left.\text { (trous } / \mathrm{cm}^{2}\right)\end{array}$ & Efficacité \\
\hline 8,2 & $6 \mathrm{~h}$ & 5,9 & 225 & 0,05 \\
\hline 8,2 & $6 \mathrm{~h} 30 \mathrm{~min}$ & 5,7 & 1221 & 0,25 \\
\hline 8,2 & $7 \mathrm{~h} 15 \mathrm{~min}$ & 5,5 & 1463 & 0,30 \\
\hline 7,2 & $5 \mathrm{~h} 30 \mathrm{~min}$ & 5,2 & 361 & 0,07 \\
\hline 7,2 & $6 \mathrm{~h}$ & 4,8 & 785 & 0,16 \\
\hline 7,2 & $6 \mathrm{~h} 30 \mathrm{~min}$ & 4,7 & 1170 & 0,24 \\
\hline
\end{tabular}




\section{A. Chambaudet, D. fellmann, P. le thanh, C. Vuilleumier}

Le maximum d'efficacité est obtenu avec un film d'épaisseur initiale $8,2 \mu \mathrm{m}$ développé pendant $7 \mathrm{~h} 15 \mathrm{~min}$ avec une solution de soude à $10 \%$, à $45^{\circ} \mathrm{C}$ avec $4 \mathrm{~V} / \mu \mathrm{m}$ et $500 \mathrm{~Hz}$. Dans ces conditions, le bruit de fond obtenu avec un film non irradié est de l'ordre de 40 trous. $\mathrm{cm}^{-2}$.

La valeur maximale de $30 \%$ d'efficacité est due au fait que les protons sont émis dans toutes les directions et pénètrent dans le LR115 avec des énergies variant de 0 à $0,58 \mathrm{MeV}$. Contrairement au résultat de comptage des traces de particules alpha dans le LR115 faisant apparaître une efficacité de comptage avoisinant $100 \%$, le maximum de $30 \%$ d'efficacité obtenu pour les protons provient du fait que toutes les traces ne traversent pas le détecteur. Par conséquent, la méthode d'analyse d'image (Quantimet 720) paraît mieux adaptée pour une meilleure efficacité de comptage.

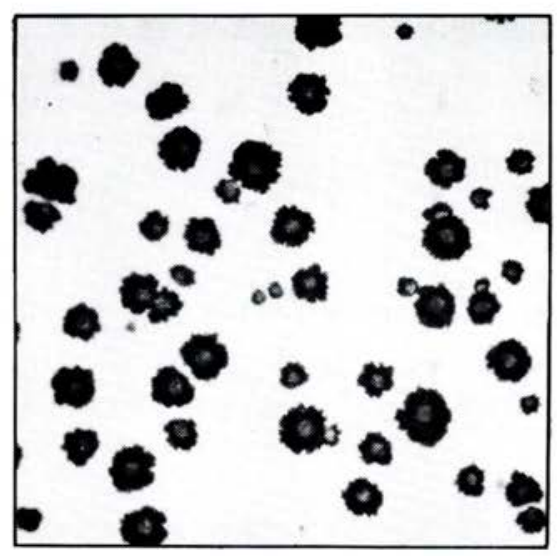

Photo 5. - Traces de protons issus de la réaction ${ }^{14} N(n, p){ }^{14} C$ dans le détecteur CR39 de $400 \mu \mathrm{m}$ développé électrochimiquement.

\section{Détection de protons avec le CR39 (photo 5)}

La mise en évidence de la détection de protons d'énergie supérieure à $60 \mathrm{keV}$ avec le CR39 a été réalisée par différents auteurs.

Nous avons procédé à divers essais de développement électrochimique ayant permis d'en établir les conditions optimales suivantes: solution de soude à $40 \%$, à $60^{\circ} \mathrm{C}$ avec un champ alternatif appliqué de $3 \mathrm{~V} / \mu \mathrm{m}$ et $500 \mathrm{~Hz}$.

Dans le souci de simplifier le matériel électronique de développement, des essais réalisés avec des paramètres identiques et une fréquence de $50 \mathrm{~Hz}$ ont fourni des résultats semblables. 


\section{Dosimétrie neutronique par détection de protons}

Le but de cette étude est de détecter les protons de recul issus de la réaction de diffusion des neutrons sur l'hydrogène contenu dans un écran de polyéthylène $\left(\mathrm{CH}_{2}\right)_{n}$ ainsi que dans les détecteurs eux-mêmes, ce qui permet d'étudier la réponse des ensembles détecteurs polymériques et convertisseurs en polyéthylène en fonction de l'énergie des neutrons incidents.

L'ensemble convertisseurs-détecteurs comporte des films de LR115 et de CR39 munis chacun d'un convertisseur de polyéthylène de $0,3 \mathrm{~mm}$ d'épaisseur et disposés entre deux écrans de cadmium permettant d'éliminer les neutrons diffusés provenant des matériaux environnants ou du sol (fig. 8).

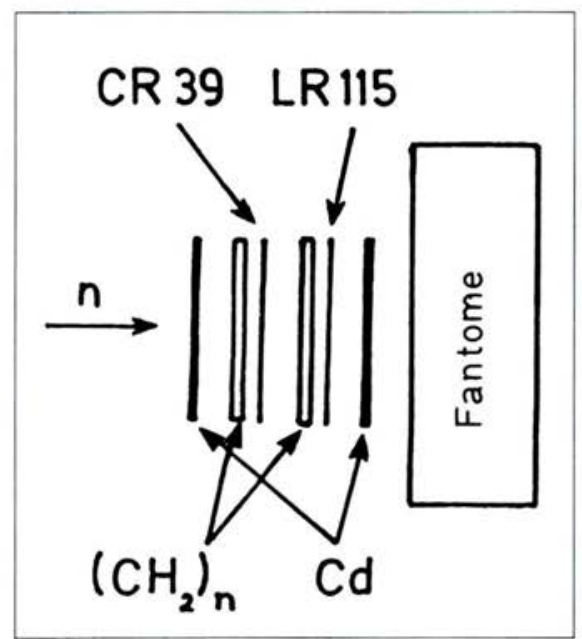

Fig. 8. - Dispositif d'irradiation aux neutrons avec convertisseurs de polyéthylène.

Pendant l'irradiation, l'ensemble convertisseurs-détecteurs est placé devant un bloc de paraffine (pour les sources radioactives) ou un réservoir d'eau (pour les neutrons monoénergétiques) jouant le rôle de fantôme; le fantôme simule la présence d'un corps humain qui rétrodiffuse les neutrons vers le détecteur lors d'une irradiation. Les neutrons monoénergétiques de 0,$06 ; 0,12 ; 0,2 ; 0,5 ; 2$ et $5 \mathrm{MeV}$ proviennent d'une réaction $(p, n)$ sur une cible de lithium ou de tritium. L'irradiation est réalisée avec l'accélérateur Van de Graaf du centre de Bruyères-le-Châtel.

La figure 9 montre la réponse par unité d'équivalent de dose obtenue avec le détecteur CR39 par Griffith et al [4], Djeffal [3], Tommasino [8] et nous-même. 


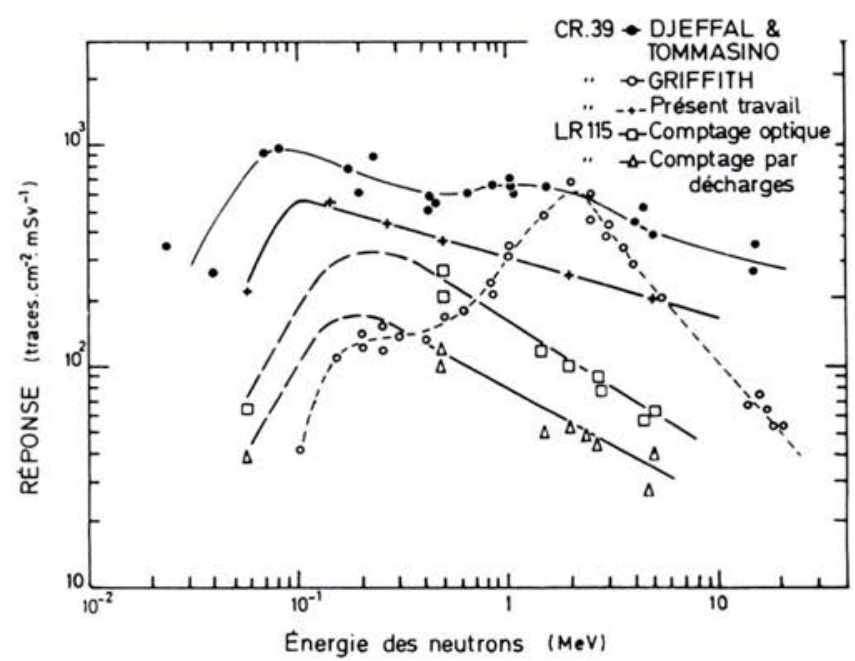

Fig. 9. - Réponse en fonction de l'énergie des neutrons du CR39 développé électrochimiquement avec prédéveloppement (- -$)$ par Griffith [4] et sans prédéveloppement $(-\bullet-)$ par Tommasino [8], Djeffal [3] et $(-+-)$ présent travail. Réponse du LR115 obtenue par comptage optique $\square$ ) et par décharges électriques $(\Delta)$.

Sur le même graphe, nos résultats avec le LR115 sont présentés en fonction de l'énergie des neutrons. Pour les différents auteurs, les conditions de développement du CR39 sont les suivantes:

- Griffith: développement électrochimique (5 heures, $2 \mathrm{kHz}, 31,5 \mathrm{kV} / \mathrm{cm}$, $6 \mathrm{~N} \mathrm{KOH}, 20^{\circ} \mathrm{C}$ ) après un développement chimique ( 5 heures, $6 \mathrm{~N} \mathrm{KOH}$, $\left.60^{\circ} \mathrm{C}\right)$.

- Djeffal, Tommasino: développement électrochimique (5 heures, $50 \mathrm{~Hz}$, $\left.30 \mathrm{kV} / \mathrm{cm}, 6 \mathrm{~N} \mathrm{KOH}, 60^{\circ} \mathrm{C}\right)$.

- Notre travail: développement électrochimique (4 h $30 \mathrm{~min}, 500 \mathrm{~Hz}$, $30 \mathrm{kV} / \mathrm{cm}, 10 \mathrm{~N} \mathrm{NaOH}, 60^{\circ} \mathrm{C}$ ).

On peut remarquer que les résultats obtenus avec le CR39 développè à $500 \mathrm{~Hz}$ sont comparables à ceux de Djeffal [3] avec une efficacité peut-être plus faible, mais qui présente l'énorme avantage d'une variation linéaire en coordonnées Log-Log excepté pour une énergie de $60 \mathrm{keV}$.

Pour le détecteur LR115 (fig. 9), nos résultats obtenus par un comptage optique et par comptage à décharges disruptives montrent une variation comparable à ceux du CR39 avec, cependant, une efficacité plus faible; en effet, les épaisseurs initiales des détecteurs CR39 et LR115 sont respectivement de $400 \mu \mathrm{m}$ et $8 \mu \mathrm{m}$ et les épaisseurs érodées par l'attaque chimique sont respectivement de 25 et $3 \mu \mathrm{m}$. 
Le tableau II donne les résultats obtenus avec les détecteurs LR115 exposés aux neutrons des sources radioactives. L'erreur relative y est portée en tenant compte seulement de l'erreur de comptage. On atteint $50 \%$ d'efficacité de comptage par la méthode de décharges disruptives après un développement électrochimique.

TABLEAU ॥

\begin{abstract}
Résultats de comptage obtenus avec les détecteurs LR115 irradiés aux neutrons des sources portables
\end{abstract}

\begin{tabular}{|c|c|c|c|c|}
\hline Source & $\begin{array}{c}\text { Energie } \\
\text { moyenne } \\
(\mathrm{MeV})\end{array}$ & $\begin{array}{l}\mathrm{R}_{0}=\text { réponse obtenue } \\
\text { par comptage optique } \\
\left(\text { traces } / \mathrm{cm}^{2}\right) /\left(10^{-2} \mathrm{mSv}\right)\end{array}$ & $\begin{array}{c}\mathrm{R}_{\mathrm{S}}=\text { réponse obtenue } \\
\text { par comptage } \\
\text { à décharges } \\
(\text { coups.cm } \\
\text { (c) }) /\left(10^{-2} \mathrm{mSv}\right)\end{array}$ & $\begin{array}{c}\text { Efficacité } \\
\mathrm{R}_{\mathrm{S}} / \mathrm{R}_{0}\end{array}$ \\
\hline AmLi & 0,5 & $1,87 \pm 10 \%$ & $0,91 \pm 4 \%$ & 0,49 \\
\hline $\mathrm{AmF}$ & 1,4 & $1,04 \pm 9 \%$ & $0,48 \pm 1 \%$ & 0,46 \\
\hline Cf-252 & 2,35 & $0,91 \pm 12 \%$ & $0,44 \pm 2 \%$ & 0,48 \\
\hline $\mathrm{AmB}$ & 2,5 & $0,76 \pm 13 \%$ & $0,41 \pm 3 \%$ & 0,54 \\
\hline $\mathrm{AmBe}$ & 4,4 & $0,55 \pm 13 \%$ & $0,24 \pm 2 \%$ & 0,44 \\
\hline
\end{tabular}

\title{
Efficacité comparée des comptages optique et automatique par analyse d'image (Quantimet 720)
}

Nous avons vu que l'épaisseur du détecteur CR39 est trop importante $(400 \mu \mathrm{m})$ pour permettre le comptage par la méthode de comptage par décharges disruptives. De ce fait, la comparaison analyse d'image-décharges disruptives n'est pas possible et la "référence" est toujours le comptage optique. Le comptage par analyse d'image (Quantimet 720) prend donc tout son intérêt pour ce détecteur étant donné que c'est la seule méthode rapide et précise. L'efficacité de comptage obtenue avec le Quantimet 720, par rapport au comptage par microscopie optique, est portée dans le tableau III. Les densités de traces du détecteur sont déterminées sur les deux faces; la face avant est exposée au faisceau incident; la face arrière est opposée à ce faisceau.

VOL. $22 \cdot \mathrm{N}^{\circ} 4$ 
A. Chambaudet, D. fellmann, P. le thanh, C. Vuilleumier

TABLEAU III

\begin{abstract}
Densités de traces obtenues avec les détecteurs CR39 irradiés aux neutrons monoénergétiques par comptage optique et comptage automatique
\end{abstract}

\begin{tabular}{|c|c|c|c|c|}
\hline \multirow{3}{*}{$\begin{array}{l}\text { Energie } \\
\text { des neutrons } \\
(\mathrm{keV})\end{array}$} & \multirow{3}{*}{$\begin{array}{l}\text { Face du } \\
\text { détecteur }\end{array}$} & \multirow{2}{*}{$\begin{array}{l}\text { Densité de } \\
\text { traces par } \\
\text { comptage } \\
\text { optique }\end{array}$} & \multirow[t]{2}{*}{$\begin{array}{l}\text { Densité de } \\
\text { traces par } \\
\text { analyseur }\end{array}$} & \multirow{3}{*}{$\frac{\text { Comptage analyseur }}{\text { Comptage optique }}$} \\
\hline & & & & \\
\hline & & \multicolumn{2}{|c|}{$\left(\right.$ traces.cm $\left.{ }^{-2}\right)$} & \\
\hline \multirow[t]{2}{*}{60} & Avant & $497 \pm 33 \%$ & $532 \pm 12 \%$ & $1,07 \pm 35 \%$ \\
\hline & Arrière & $144 \pm 68 \%$ & $168 \pm 23 \%$ & $1,167 \pm 72 \%$ \\
\hline \multirow[t]{2}{*}{500} & Avant & $7462 \pm 6 \%$ & $5468 \pm 4 \%$ & $0,73 \pm 7 \%$ \\
\hline & Arrière & $5850 \pm 8 \%$ & $5124 \pm 3 \%$ & $0,88 \pm 9 \%$ \\
\hline \multirow[t]{2}{*}{2000} & Avant & $5300 \pm 8 \%$ & $4192 \pm 1 \%$ & $0,79 \pm 8 \%$ \\
\hline & Arrière & $3562 \pm 11 \%$ & $2937 \pm 2 \%$ & $0,824 \pm 11 \%$ \\
\hline \multirow[t]{2}{*}{5000} & Avant & $4265 \pm 8 \%$ & $2832 \pm 7 \%$ & $0,66 \pm 11 \%$ \\
\hline & Arrière & $2758 \pm 12 \%$ & $2110 \pm 8 \%$ & $0,77 \pm 14 \%$ \\
\hline
\end{tabular}

II apparaît que jusqu'à une valeur de densité de l'ordre de 3500 traces $/ \mathrm{cm}^{2}$, l'efficacité de comptage par analyse d'image est d'environ $90 \%$. Pour des densités de traces plus élevées, l'efficacité diminue. Ceci est dû au recouvrement des traces entre elles qui devient très important et surtout très irrégulier. Pour limiter ce phénomène, il conviendra de réduire la taille des traces $(>50 \mu \mathrm{m})$ en intervenant sur les paramètres du développement électrochimique.

\title{
CONCLUSION
}

L'étude réalisée a mis en évidence la possibilité de réaliser un dosimètre neutronique par réaction $(n, p)$ avec des ensembles de détecteurs LR115 et CR39 munis de convertisseurs de polyéthylène. La réponse par unité d'équivalent de dose décroît lentement avec l'énergie croissante des neutrons. Le détecteur LR115 présente l'avantage de permettre un développement électrochimique des traces suivi d'un comptage par la méthode à décharges disruptives.

Le détecteur CR39 permet, lui aussi, le développement électrochimique, mais du fait de son épaisseur importante $(400 \mu \mathrm{m})$, le comptage des traces ne peut se faire que par analyse d'image. 
La rapidité et la précision de comptage permet, par analyse d'image ou par décharges électriques, d'envisager l'utilisation en routine de ces détecteurs de traces nucléaires dans la dosimétrie neutronique.

La suite de cette étude portera sur la possibilité d'obtenir une réponse constante des détecteurs en fonction de l'énergie des neutrons incidents avec la meilleure efficacité possible de comptage.

\section{REMERCIEMENTS}

Nous remercions le professeur Bugnon du Centre hospitalier et universitaire de Besançon qui a permis les travaux sur le Quantimet de son laboratoire (cytologie et cytogénétique).

\section{RÉFÉRENCES BIBLIOGRAPHIQUES}

[1] DECOSSAS J.L. Endommagement des dérivés cellulosiques par des ions. Modélisation de la trace. Détection des protons. Application à la dosimétrie neutronique. Thèse $\mathrm{n}^{\circ}$ 12-82, Université de Limoges, 1982.

[2] DECOSSAS J.L., VAREILLES J.C., MOLITON J.P., TEYSSIER J.L. Theoretical study and calculation of the response of a fast neutron dosimeter based on track detection. Radiat. Prot. Dosim., 1983, 5, 163-170.

[3] DJEFFAL S. Dosimétrie personnelle des neutrons rapides à l'aide d'une nouvelle procédure de développement électrochimique du CR39. Thèse de magister en génie nucléaire, CEN Alger, 1984.

[4] GRIFFITH R.V., THORNGATE J.H., DAVIDSON K.J., RUEPPEL D.W., FISHER J.C., TOMMASINO L., ZAPPAROLLI G. Monoenergetic neutron response of selected etch plastics for personnel neutron dosimetry. Radiat. Prot. Dosim., 1981, 1 (1), 61-71.

[5] KIEFER H., MAUSHART R. Radiation protection measurement. Oxford: Pergamon Press, 1972.

[6] LE THANH P., NIKPAY P. Un dispositif de comptage à décharges disruptives permettant d'atténuer les décharges disruptives multiples. In: Solid state nuclear track detectors, Lyons, 2-6 July 1979 (FRANÇOIS M. Ed.). Oxford: Pergamon press, 1980, 337-361.

[7] LUCK H.B. Diameter evolution of proton tracks in a cellulose nitrate detector. Nucl. Instrum. Meth., 1974, 116, 613-614.

[8] TOMMASINO L., ZAPPAROLLI G., SPIEZIA P., GRIFFITH R.V., ESPINOSA G. Different etching processes of damage track detectors for personnel neutron dosimetry. Nucl. Tracks, 1984, 8 (1/4), 335-339. 